\title{
THE EXISTENCE OF WIDELY CONNECTED AND BICONNECTED SEMIGROUPS
}

\author{
PAUL M. SWINGLE
}

A. D. Wallace in [10] and Wallace and R. J. Koch in Theorem 3 of $[3$, p. 683] have shown that if a topological semigroup with unit is a metric indecomposable continuum then it is a topological group: it is known that a solenoid is both a compact connected group and an indecomposable continuum. We have shown [5] that any compact indecomposable continuum $I$ contains a widely connected subset $W$ such that $\bar{W}=I$ : below it is shown that if $I$ is also a topological group with unit and other stated simple properties, then $W$ can be taken so that it is a widely connected topological group. Here $W$ is nonbicompact and it nowhere contains an arc in contrast to the well known result by Pontrjagin that a bicompact connected topological group does contain an arc. Koch in [2] has shown recently that a bicompact connected topological semigroup with unit contains an arc; in contrast to this we also give below an example of a biconnected topological semigroup: it is nonbicompact, it has a unit and also a dispersion point at its zero.

To show the existence of both of these we make strong use of Zermelo's well ordering theorem and assume the hypothesis of the continuum. The existence proof is a modification of the well known one used by Knaster and Kuratowski in [1, pp. 241, 250] to show the existence of a biconnected set. It is a modification of this by Wilder, which does not make a strong use of Zermelo's well ordering theorem, which we use in [5] to show the existence of a widely connected set: however we are unable to use this below because the construction must give also the desired algebraic properties.

We recall the following definitions. The connected set $W$ is widely connected if and only if every nondegenerate connected subset $C$ of $W$ has the same closure that $W$ does, i.e. $\bar{C}=\bar{W}$. The connected set $B$ is biconnected if and only if it is not the union of two disjoint nondegenerate connected subsets; $d \in B$ is a dispersion point of $B$ if $B-d$ is totally disconnected. For more basic topological definitions see Moore in [4] or Wilder in [11]; bicompact is as in [11, p. 34]. For theorems on indecomposable continua see Moore in [4, pp. 75-77]. We denote the null set by $\varnothing$. For basic semigroup definitions see Wallace in [9].

Presented to the Society, August 27, 1958 under the title Widely connected algebras; received by the editors September 16, 1958 and, in revised form, May 20, 1959. 
A topological semigroup $S$ is a Hausdorff space together with a continuous associative operation. Let $I$ as below be a metric indecomposable continuum and also a topological semigroup with unit. We call the associative operation on $I$ a multiplication and multiplication by an inverse a division, using symbols $\cdot$ and $\div$ for these. By Gen $(p)$, we mean the set of all elements of $I$ which can be built up by multiplications and divisions by $p \in I$, i.e. with $p$ as generator: obviously Gen $(p)$ contains $p$, its inverse and the unit if the inverse exists. Similarly Gen $(P)$ means the set built up by these operations with $P$ as generating set. Below we will choose a class $\left\{p_{\alpha}\right\}$ of elements of $I$. We take $P_{1}=\operatorname{Gen}\left(p_{1}\right)$ and $P_{\alpha}=\operatorname{Gen}\left(\left(U P_{\beta}\right) \cup_{p_{\alpha}}\right)$ for $(\beta<\alpha)$ where $\beta$ and $\alpha$ are ordinals.

We need the following Algebraic Assumption, denoted by (*AA) below: Let $g$ and $G$ each be a product of a finite number of elements of $I$; let $H(p, G)$ be a similar product including $n$ occurrences of $p \in I$ and $n^{\prime}$ occurrences of the inverse of $p$ : then we assume that there exist only a countable number of solutions $p$ of $H(p, G)=g$.

Lemma 1. If $\left\{p_{\alpha}\right\}$ is a countable class, $p_{\alpha} \in I$, then Gen $\left(U_{p_{\alpha}}\right)$ is also countable, i.e. the set generated by $\cup_{p_{\alpha}}$ is countable.

The proof is elementary.

Lemma 2. Let I be a compact indecomposable continuum which is a topological semigroup and $\left({ }^{*} A A\right)$ holds; let also $P$ and $Q$ be countable subsets of $I$ such that $\operatorname{Gen}(P)$ and $\operatorname{Gen}(Q)$ are disjoint. Then there exists $p \in I$ such that $p$ is not contained in any composant of $I$ which contains a point of Gen $(P) \cup \operatorname{Gen}(Q)$ and Gen $(P \cup p)$ and Gen $(Q)$ are disjoint.

Proof. Let $p \in I$. Let $F(p, P)$ be any element of Gen $(P \cup p)$ and $H$ be any element of Gen $(Q)$. The equation $F(p, P)=H$ may involve $n^{\prime}$ occurrences of the inverse of $p$ and say $n, \geqq 1$, occurrences of $p$ itself. By our Algebraic Assumption ( $\left.{ }^{*} \mathrm{AA}\right)$ above it has at most a countable number of solutions $p$. By an argument similar to that of Lemma 1 with $n$ and $n^{\prime}$ fixed there are only a countable number of possible equations of type $F(p, P)=H$. This is then also true when one varies $n$ and $n^{\prime}$. Thus there exist but a countable number of solutions $p$ of all possible equations of type $F(p, P)=H$.

By Lemma 1 we see that Gen $(P)$ and Gen $(Q)$ are both countable. Hence Gen $(P) \cup \operatorname{Gen}(Q)$ is contained in the union of a countable number of composants of $I$ and $I$ has uncountably many disjoint composants $[4, p .77]$. Thus $p$ can be chosen in a new composant of $I$ and so that it is not the solution of an equation of type $F(p, P)$ 
$=H$ above. Therefore $p$ can be chosen as desired in the lemma such that Gen $(P \cup p)$ and Gen $(Q)$ are disjoint.

Lemma 3. Let $I, P$ and $Q$ be as in Lemma 2. Let $T$ be a perfect subset of $I$. Then there exists $q \in T$ such that $\operatorname{Gen}(P)$ and $\operatorname{Gen}(Q \cup q)$ are disjoint.

Proof. Let $q \in T \subset I$. Let $F(q, Q)$ be any element of Gen $(Q \cup q)$ and $H$ be any element of Gen $(P)$. Then as in the argument in the proof of Lemma 2 we conclude that there exists but a countable number of solutions $q$ of all the possible equations of type $F(q, Q)=H$. By Lemma 1 , Gen $(Q) \cup \operatorname{Gen}(P)$ is countable and $T$ is known to be uncountable. Therefore $q \in T$ can be taken so that it is not contained in Gen $(Q) \cup \operatorname{Gen}(P)$ and is not a solution of an equation of type $F(q, Q)=H$. Hence Gen $(P)$ and $\operatorname{Gen}(Q \cup q)$ are disjoint and the lemma is true.

We need the following. We say that $B$ is a separating boundary of a set $I$ if and only if $B$ is the common boundary in $I$ of two disjoint non-null open subsets of $I$. The construction of the widely connected set $S$ below is based upon Knaster and Kuratowski's well known Theorem 37 of $[1$, p. 233] for an $n$-dimensional metric space, $n \geqq 2$, stating that $S \subset I$ is disconnected if and only if there exists a separating boundary $B$ of $S$ such that $I-S \supset B$, i.e. $S \cap B=\varnothing$. We denote the class of separating boundaries of $I$ by $\left\{B_{\alpha}\right\}$, noting that $I \supset \cup B_{\alpha}$. We denote the class of perfect subsets of $I$ by $\left\{T_{\alpha}\right\}$. As noted in $[1$, pp. 248-251] both $\left\{B_{\alpha}\right\}$ and $\left\{T_{\alpha}\right\}$ have the power $2 \aleph_{0}$ of the linear continuum under our assumptions. We note that each $T_{\alpha}$ is of the power of the linear continuum; also each $B_{\alpha}$ is, since $I$ contains uncountably many disjoint composants each dense in $I$, disregarding the trivial $I$ degenerate case.

THEOREM 1. Let I be a compact metric indecomposable continuum which is a topological semigroup with unit; let also $\left({ }^{*} A A\right)$ be true and each proper subcontinuum of $I$ be a simple continuous arc. Then I contains a widely connected topological semigroup $S$ with unit: $\bar{S}=I$.

PROof. Let $\Omega$ be the first transfinite ordinal whose cardinal is that of the linear continuum, i.e. is $2 \aleph_{0}$. We follow Knaster and Kuratowski's well known construction in [1, pp. 248-251] well ordering each of the classes $\left\{B_{\alpha}\right\}$ and $\left\{T_{\alpha}\right\}$ as follows:

$$
\begin{array}{ll}
B_{1}, B_{2}, \cdots ; B_{\omega}, \cdots ; \cdots ; \cdots, B_{\alpha}, \cdots & \alpha<\Omega, \\
T_{1}, T_{2}, \cdots ; T_{\omega}, \cdots ; \cdots ; \cdots, T_{\alpha}, \cdots & \alpha<\Omega .
\end{array}
$$

We now use Lemmas 2 and 3 alternately to choose points $p_{\alpha}$ and $q_{\alpha}$. 
The set $U_{p_{\alpha}}$ will be widely connected, but we need for closure in multiplication more points than that, i.e. we need Gen $\left(U_{p_{\alpha}}\right)$ : we have no control over where the points Gen $\left(U_{p_{\alpha}}\right)-U_{p_{\alpha}}$ may occur and it is this fact which adds complexity to our construction. However Lemmas 2 and 3, and the countability given by Lemma 1 and the hypothesis of the continuum, enables us to choose the $p_{\alpha}$ and $q_{\alpha}$ so that Gen $\left(\cup_{p_{\alpha}}\right)$ and Gen $\left(U_{q_{\alpha}}\right)$ are disjoint: this permits us to prove the theorem.

Choose $p_{1} \in B_{1}$ and let $P_{1}=$ Gen $\left(p_{1}:\right)$. Let in Lemma $3 P=p_{1}$ and $Q=\varnothing$. Then we can choose $q_{1} \in T_{1}$ such that $P_{1}$ and Gen $\left(Q \cup q_{1}\right),=Q_{1}$ say, are disjoint. Now using Lemma 2 we can choose $p_{2} \in B_{2}-P_{1}-Q_{1}$ such that Gen $\left(P_{1} \cup p_{2}\right),=P_{2}$ say, and Gen $\left(Q_{1}\right)$ are disjoint; we note this last fact lets us say merely choose $p_{2} \in B_{2}$; also $p_{2} \in a$ composant of $I$ which does not contain a point of Gen $\left(P_{1}\right) \cup$ Gen $\left(Q_{1}\right)$. We follow this by choosing $q_{2} \in T_{2}-P_{2}-Q_{1}$, i.e. $p_{2} \in T_{2}$, using Lemma 3 , such that Gen $\left(P_{2}\right)$ and Gen $\left(Q_{1} \cup q_{2}\right)$ are disjoint.

We continue this process of choosing $p_{\alpha}$ and $q_{\alpha}$ for $(\alpha<\Omega)$ by transfinite induction. Consider the case where we choose $p_{\beta}$ and $q_{\beta}$, where, for all $(\alpha<\beta), p_{\alpha}$ and $q_{\alpha}$ already have been chosen. We note that $Q_{\beta}=\operatorname{Gen}\left(\cup q_{\alpha}\right)$ and $P_{\beta}=\operatorname{Gen}\left(\left(\cup_{p_{\alpha}}\right) \cup p_{\beta}\right)$; also note that Gen $\left(\cup q_{\alpha}\right)$ $=\mathrm{Gen}\left(\cup Q_{\alpha}\right)$ so that we need not distinguish between these. Thus using Lemma 2 we choose $p_{\beta} \in B_{\beta}$ such that Gen $\left(\cup Q_{\alpha}\right)$ and Gen $\left(p_{\beta} \cup\left(\cup p_{\alpha}\right)\right)$ are disjoint and $p_{\beta}$ is in no composant of $I$ which contains a point of Gen $\left(\cup_{p_{\alpha}}\right) \cup \operatorname{Gen}\left(\cup Q_{\alpha}\right)$. Then $q_{\beta} \in T_{\beta}$ is chosen according to Lemma 3 . This process is possible because at any step Gen $\left(U_{p_{\alpha}}\right) \cup$ Gen $\left(U q_{\alpha}\right)$ is countable by Lemma 1 and the hypothesis of the continuum, while both $B_{\beta}$ and $T_{\beta}$ are uncountable sets.

Let $S=$ Gen $\left(\cup_{p_{\alpha}}\right)$, = Gen $\left(U P_{\alpha}\right)$, for $(\alpha<\Omega): S$ is our desired widely connected topological semigroup as we now show. By Knaster and Kuratowski's Theorem 37 above we see that $S$ is connected because for each separating boundary $B_{\alpha}$ of $I$, and so of $S$, we have $p_{\alpha} \in S$, i.e. $B_{\alpha} \cap S \neq \varnothing$. We note that each neighborhood in our metric $I$ had a $B_{\alpha}$ as boundary and so $\bar{S}=I$, i.e. $S$ is dense in $I$. Suppose that $S$ is not widely connected, i.e. that $S$ contains a nondegenerate connected subset $C$ such that $\bar{S} \neq \bar{C}$. Then $\bar{C} \subset I=\bar{S}$ is a proper subcontinuum of $I$ and so, by our hypothesis in Theorem $1, \bar{C}$ is a simple continuous arc. Hence $\bar{C} \supset a T_{\alpha}$; thus the nondegenerate connected set $C, \subset S$, contains an arc and so a $T_{\beta}$, although from our proof $q_{\beta}$ is not an element of Gen $\left(\mathrm{U}_{p_{\alpha}}\right)=S$. Therefore $S$ must be widely connected.

We have constructed an ascending tower of semigroups $P_{1} \subset P_{2}$ $\subset \cdots \subset P_{\alpha} \subset \cdots$ for $(\alpha<\Omega)$ above. If $p, q \in \bigcup P_{\alpha}$, then $p, q \in$ some 
$P_{\alpha}$ and so $p q \in P_{\alpha} \subset S$. Hence $S$ is a semigroup inheriting the associative multiplication property from $I$ : that its multiplication is continuous is also an inheritance from $I$. Hence $S$ is a widely connected topological semigroup and so the Theorem is true, noting that the unit $\in \operatorname{Gen}\left(p_{1}\right) \subset S$.

CoRollary 1. Let I be as in Theorem 1. Then I contains a widely connected topological group $S$ and $\bar{S}=I$.

Proof. As noted above Koch and Wallace have shown that $I$ is a topological group. Hence, if $p \in I$, its inverse exists: thus each $p \in S \subset I$ of Theorem 1 has its inverse. Similarly we note that $S$ inherits all the properties from $I$ which it needs in order to be a topological group. Thus the corollary is true.

We remark: If $I$ had a more complicated algebra involving both - and + then Lemmas 1, 2 and 3 go through for Gen $(P)$ redefined by using $+-\cdot \div$; thus we might be able to prove that $I$ contains densely a widely connected subset which inherits this algebra. See perhaps [7, pp. 140-141]; if the example there is true, it is nonbicompact by [2].

We need the following in order to show the existence of a biconnected topological semigroup. Let $N=\{x \mid 0 \leqq x \leqq 1\}$ and $C$ be the Cantor ternary set on $N$. Let $I$ be the cartesian product $C \times N$, with however $(c, 0)$ the same point $(0,0)$ for all $c \in C$. Let $(c, x) \cdot\left(c^{\prime}, x^{\prime}\right)$ $=\left(c \cdot c^{\prime}, x \cdot x^{\prime}\right)$ for $c, c^{\prime} \in C$ and $x, x^{\prime} \in X$, where $c \cdot c^{\prime}$ can be taken so that $x \cdot x^{\prime}$ is by the ordinary multiplication of real numbers. Then $I$ is known to be a topological semigroup; also $I$ is one of the sets used by Knaster and Kuratowski in [1, pp. 241, 250] to construct a biconnected subset $S$ with dispersion point $(0,0)$, and dense in $I$. Thus we can use $I$ to obtain the following:

Theorem 2. If $\left({ }^{*} A A\right)$ is true in I above, then there exists a biconnected topological semigroup $S$ with a unit and with a dispersion point at $(0,0)$ where $\bar{S}=I$.

Proof. We state (*AA) for this new $I$ and make similar changes in Lemmas 1, 2 and 3. We use the method of the proof of Theorem 1, taking however, each $B_{\alpha}$ not containing $(0,0)$. Following Knaster and Kuratowski in [1, pp. 241,250] there is no difficulty in seeing that a similar construction to that used for Theorem 1 above gives a biconnected topological semigroup $S$, dense in $I$, with $(0,0)$ as its dispersion point and with $(1,1)$ as unit.

Because of the length of the above we give the properties we obtain of biconnected semigroups and widely connected semigroups in [6]. 


\section{REFERENCES}

1. B. Knaster and C. Kuratowski, Sur les ensembles connexes, Fund. Math. vol. 2 (1921) pp. 206-253.

2. R. J. Koch, Arcs in partially ordered spaces, to appear.

3. R. J. Koch and A. D. Wallace, Maximal ideals in compact semigroups, Duke Math. J. vol. 21 (1954) pp. 681-685.

4. R. L. Moore, Foundations of point set theory, Amer. Math. Soc. Colloquium Publications, vol. 13, 1932.

5 P. M. Swingle, Two types of connected sets, Bull. Amer. Math. Soc. vol. 37 (1931) pp. 254-258.

6. - Widely connected and biconnected semigroups, Proc. Amer. Math. Soc. vol. 11 (1960) pp. 249-254.

7. Summer Institute on Set Theoretic Topology, Madison, 1955.

8. A. D. Wallace and R. J. Koch, Notes on mobs, mimeographed, 1956, Tulane University.

9. A. D. Wallace, The structures of topological semigroups, Bull. Amer. Math. Soc. vol. 61 (1955) pp. 95-112.

10. - Indecomposable semigroups, Math. J. Okayama Univ. vol. 3 (1953) pp. 1-3.

11. R. L. Wilder, Topology of manifolds, Amer. Math. Soc. Colloquium Publications, vol. 32, 1948.

The University of Miami 